DE

M E D I C I N A

T R O P I C A L

$\mathrm{DE}$

SÃO PAULO

JOURNAL OF THE SÃO PAULO INSTITUTE OF TROPICAL MEDICINE

(1) Universidade de São Paulo, Faculdade de Odontologia, Departamento de Estomatologia, São Paulo, São Paulo, Brazil

(2) Instituto Adolfo Lutz, Núcleo de Ciências Biomédicas, Bauru, São Paulo, Brazil

(3) Universidade de São Paulo, Instituto de Medicina Tropical de São Paulo, São Paulo, São Paulo, Brazil

(4)Universidade Federal de São Paulo, Laboratório Especial de Micologia, São Paulo, São Paulo, Brazil

Correspondence to: Carina Domaneschi. Universidade de São Paulo, Faculdade de Odontologia, Departamento de Estomatologia, Av. Prof. Lineu Prestes, 2227, CEP 05508-000, São Paulo, SP, Brazil. Tel: +55 11 3091-7893,

Fax: $+55113091-7820$

E-mail: domaneschi@usp.br

Received: 31 March 2016

Accepted: 14 February 2017

\section{Phenotypic and genotypic detection of Candida albicans and Candida dubliniensis strains isolated from oral mucosa of AIDS pediatric patients}

Harisson Oliveira Livério1, Luciana da Silva Ruiz², Roseli Santos de Freitas ${ }^{3}$, Angela Nishikaku ${ }^{4}$, Ana Clara de Souza1, Claudete Rodrigues Paula ${ }^{1}$, Carina Domaneschi ${ }^{1}$

\section{ABSTRACT}

The aim of this study was to assess a collection of yeasts to verify the presence of Candida dubliniensis among strains isolated from the oral mucosa of AIDS pediatric patients which were initially characterized as Candida albicans by the traditional phenotypic method, as well as to evaluate the main phenotypic methods used in the discrimination between the two species and confirm the identification through genotypic techniques, i.e., DNA sequencing. Twenty-nine samples of $C$. albicans isolated from this population and kept in a fungi collection were evaluated and re-characterized. In order to differentiate the two species, phenotypic tests (Thermotolerance tests, Chromogenic medium, Staib agar, Tobacco agar, Hypertonic medium) were performed and genotypic techniques using DNA sequencing were employed for confirmation of isolated species. Susceptibility and specificity were calculated for each test. No phenotypic test alone was sufficient to provide definitive identification of $C$. dubliniensis or $C$. albicans, as opposed to results of molecular tests. After amplification and sequencing of specific regions of the 29 studied strains, $93.1 \%$ of the isolates were identified as $C$. albicans and $6.9 \%$ as $C$. dubliniensis. The Staib agar assay showed a higher susceptibility $(96.3 \%)$ in comparison with other phenotypic techniques. Therefore, genotypic methods are indispensable for the conclusive identification and differentiation between these species.

KEYWORDS: Candida dubliniensis. Candida albicans. Children. HIV/AIDS Phenotypic and genotypic methods.

\section{INTRODUCTION}

Oral candidosis is the most frequent manifestation in Human Immunodeficiency Virus (HIV)-infected children, with prevalence varying from $20 \%$ to $72 \%$. Infection usually persists for a long time, it is punctuated by periods of remission, and is frequently resistant to antifungal conventional therapies. These manifestations may also occur in healthy infants in the first six months of life ${ }^{1}$.

Oral colonization by Candida spp. has been thoroughly investigated in HIV-positive adults, but the number of studies with HIV-positive children is still small $^{2}$.

Candida albicans is the most frequent species related to oral fungal infections in adults and children and it is the most virulent species of the genus. However, Candida dubliniensis, a species recently described by Sullivan et al. ${ }^{3}$, has attracted considerable attention due to its almost exclusive association with HIV+/ AIDS individuals. Candida dubliniensis has been isolated from erythematosus candidosis, mainly 
from the oropharyngeal mucosa of HIV+/AIDS patients ${ }^{3,4}$. Nevertheless, few studies on Candida dubliniensis infection among the pediatric population have been conducted so $\mathrm{far}^{5,6}$. Al-Ahmad et al. ${ }^{7}$ studied $C$. dubliniensis in cariesfree and caries-active healthy children in relation to the oral microbiota and found an association between caries-active children and the presence of $C$. dubliniensis.

Since its characterization in 1995, C. dubliniensis has attracted the attention of researchers because this yeast presents similar phenotypic and genotypic characteristics to C. albicans. C. dubliniensis differs from C. albicans with respect to frequency of isolation, pathogenic characteristics, and resistance to antifungal drugs, as well as by presenting higher capacity of adherence to oral mucosa cells ${ }^{8}$. The similarities between these two species hinder their prompt differentiation and may lead to results that underestimate their prevalence.

Phenotypic tests are useful for presumptive identification of $C$. dubliniensis, but they do not provide definitive identification; in contrast, molecular methods provide conclusive identification, but they are difficult to perform, expensive, and require specific equipment ${ }^{9}$.

Definitive identification of $C$. dubliniensis is still a problem in routine laboratories because phenotypic and genotypic characteristics of isolated samples are indispensable for final characterization. Reports on the incidence of this yeast by reference laboratories are needed for a better understanding of the epidemiology of this new species $^{10}$. Most importantly, strains of $C$. dubliniensis present higher chance of developing antifungal resistance to "azoles" in comparison with strains of $C$. albicans. Moreover, a phenotype resistant to fluconazole can be induced in vitro after exposure to the drug ${ }^{11}$.

Considering the limited number of studies among the pediatric population and the need to establish methods for the prompt identification of $C$. dubliniensis, which may contribute to an efficient therapy, the aim of this study was to assess a collection of yeasts to verify the presence of $C$. dubliniensis among strains isolated from the oral mucosa of AIDS pediatric patients which were initially characterized as $C$. albicans by the traditional phenotypic method, as well as to evaluate the main phenotypic methods used in the discrimination between the two species and confirm the identification through genotypic techniques, i.e., DNA sequencing.

\section{MATERIALS AND METHODS}

\section{Samples and Patients}

Twenty-nine strains from collections were included in this study. They had been previously identified as $C$. albicans through the traditional phenotypic method, isolated from the oral mucosa of AIDS pediatric patients in the Infectious Diseases outpatient clinic and daycare hospital of the Children Institute, Hospital das Clínicas, São Paulo, Brazil. Strains belong to the fungi collection of the Laboratory of Pathogenic Yeasts, Biomedical Science Institute II, University of São Paulo (USP), and were kept lyophilized and in mineral oil. The research was approved by the Research Ethics Committee of USP in accordance with the Declaration of Helsinki (1975).

\section{Phenotypic re-characterization}

Yeasts were studied according to the traditional phenotypic method described by Kurtzman et al. ${ }^{12}$.

\section{Quality control}

Control strains ATCC64548 (C. albicans) and 777 (C. dubliniensis) were included in all tests performed in this study.

Differentiation between C. albicans and C. dubliniensis

\section{Phenotypic Techniques}

\section{Thermotolerance}

All the C. albicans strains were cultivated in Sabouraud dextrose agar (Difco- Detroit, EUA) to test their ability to grow at $45^{\circ} \mathrm{C}$. Yeast growth under these conditions indicates the presence of $C$. albicans, whereas the absence of growth indicates presence $C$. dubliniensis ${ }^{13}$.

\section{Chromogenic medium}

Strains were seeded in CHROMagar Candida medium (Microbiology, EUA) aiming to obtain specific coloring for the two species according to the manufacturer: light green for $C$. albicans and dark green for C. dubliniensis.

\section{Microculture in Staib Agar}

Microcultures in Staib Agar were prepared according to Staib and Morschhauser ${ }^{14}$. Readings were performed under optical microscope and the presence of clamydoconidium in bunches would lead to $C$. dubliniensis.

\section{Culture in Tobacco agar}

Culture in tobacco agar was conducted according to Khan et al. ${ }^{15}$. Microscopic examination would show the development of clamydoconidiums and hyphae, typical of C. dubliniensis. 
Table 1 - Phenotypic and genotypic identification of isolates previously identified through phenotypic methods as $C$. albicans

\begin{tabular}{|c|c|c|c|c|c|c|}
\hline \multirow[b]{2}{*}{ Species (n) } & \multicolumn{5}{|c|}{ Phenotypic Identification } & \multirow{2}{*}{$\begin{array}{l}\text { Genotypic } \\
\text { Identification }\end{array}$} \\
\hline & $\begin{array}{c}\text { Temperature } 45 \\
{ }^{\circ} \mathrm{C}\end{array}$ & $\begin{array}{l}\text { Chromogenic } \\
\text { Medium }\end{array}$ & Staib agar & Tobacco agar & $\begin{array}{l}\text { Hypertonic } \\
\text { Medium }\end{array}$ & \\
\hline C. albicans & 24 & 25 & 27 & 23 & 24 & 27 \\
\hline C. dubliniensis & 05 & 04 & 02 & 06 & 05 & 02 \\
\hline
\end{tabular}

\section{Hypertonic Broth}

Isolates were seeded in hypertonic Sabouraud broth. The growth in this broth would suggest the presence of Candida albicans strain because $C$. dubliniensis does not grow in medium with such high concentration of $\mathrm{NaCl}^{16}$.

\section{Genotypic Technique}

The genotypic technique was used as a standard in the identification of Candida albicans and dubliniensis. DNA extraction of the total yeasts was performed according to the commercial protocol PrepManTM Ultra Sample Preparation reagent Quick Reference Card (Applied Biosystems, EUA). Amplification was performed using primers that amplify the fragments of the ITS region of the rDNA $^{17}$. For the sequencing of the ITS region, the forward ITS1 (5'-TCCGTAGGTGAACCTGCGG-3') and reverse ITS4 (5'-TCCTCCGCTTATTGATAT GC-3') primers $^{18}$ were also used. The sequencing agreement was obtained by comparison with sequences available in GenBank with the aid of the BLASTn software.

\section{Statistical analysis}

Susceptibility and specificity measures were used to evaluate the performance of classification of Thermotolerance tests, Chromogenic medium, Staib agar, Tobacco agar, and Hypertonic medium. In this analysis, DNA sequencing was considered as gold standard. The tests were used to classify samples of C. albicans and C. dubliniensis.

\section{RESULTS}

According to traditional methods of identification previously performed, all the strains studied were $C$. albicans.

Table 1 describes the identification suggested by phenotypic markers used in this study for differentiation between $C$. albicans and $C$. dubliniensis. After amplification and sequencing of specific regions of the 29 studied strains, $27(93.1 \%)$ of the isolates were identified as C. albicans and two (6.9\%) as C. dubliniensis.

Table 2 shows the performance measures of susceptibility and specificity testing of $C$. albicans and $C$. dubliniensis.

The phenotypic characteristics observed in the two samples molecularly identified as $C$. dubliniensis are described in Table 3.

Table 2 - Performance of susceptibility and specificity testing to $C$. albicans and $C$. dubliniensis

\begin{tabular}{lcc}
\hline Test & Susceptibility & Specificity \\
\hline Thermotolerance & $81.5 \%$ & $0.0 \%$ \\
Chromogenic medium & $88.9 \%$ & $50.0 \%$ \\
Staib agar & $96.3 \%$ & $50.0 \%$ \\
Tobacco agar & $81.5 \%$ & $50.0 \%$ \\
Hypertonic medium & $88.9 \%$ & $100.0 \%$ \\
\hline
\end{tabular}

\section{DISCUSSION}

The prevalence of $C$. dubliniensis has been widely discussed in the specific literature worldwide with varied results. Research on the incidence of $C$. dubliniensis is needed for a better understanding of its epidemiology, mainly in South America where its frequency is not wellknown ${ }^{10}$.

Table 3 - Phenotypic characteristics of the two strains molecularly identified as C. dubliniensis

\begin{tabular}{|c|c|c|c|c|c|}
\hline \multirow{2}{*}{$\begin{array}{l}\text { Genotypic } \\
\text { Identification }\end{array}$} & \multicolumn{5}{|c|}{ Phenotypic Identification } \\
\hline & Temperature $45^{\circ} \mathrm{C}$ & $\begin{array}{l}\text { Chromogenic } \\
\text { medium }\end{array}$ & Staib agar & Tobacco agar & Hypertonic medium \\
\hline C. dubliniensis & Presence of growth & Light-green & $\begin{array}{c}\text { Presence of } \\
\text { clamydoconidiums }\end{array}$ & $\begin{array}{c}\text { Absence of } \\
\text { clamydoconidium }\end{array}$ & Absence of growth \\
\hline C. dubliniensis & Presence of growth & Dark-green & $\begin{array}{c}\text { Absence of } \\
\text { clamydoconidium }\end{array}$ & $\begin{array}{c}\text { Presence of } \\
\text { clamydoconidiums }\end{array}$ & Absence of growth \\
\hline
\end{tabular}


In this area, the prevalence of $C$. dubliniensis isolates seems to be lower than the that found in European countries or in the USA ${ }^{19,20}$. However, in Argentina, C. dubliniensis was found in $12.9 \%$ of the oropharyngeal isolates of HIVpositive patients ${ }^{21}$. Authors consider their results similar to those found in Ireland (18-32\%), but different from those in Brazil (2.8\%), where two (5.4\%) isolates of $C$. dubliniensis were identified among 37 strains isolated from the oral erythematous candidiasis from HIV-positive and HIV-negative patients previously identified as C. albicans ${ }^{10}$. Our findings confirm the data found in the literature. Two $(6.9 \%)$ out of a total of 29 strains of the oral mucosa initially identified as $C$. albicans were genotypically characterized as $C$. dubliniensis.

In the present study, we consider that rapid identification of $C$. dubliniensis is relevant because it allows professionals to choose the most appropriate medicine to treat patients, as the species proved resistant to fluconazole.

Routine discrimination between $C$. albicans and C. dubliniensis has been a problem in microbiological diagnostic laboratories and the description of phenotypic tests for differentiation between these two species in the literature has increased in the past ten years ${ }^{22}$. Phenotypic tests provide a suggestive identification of the focal species according to its behavior under a given set of conditions. However, although these methods are simple and inexpensive, they are normally time-consuming and/ or demonstrate, in most cases, low ability to discriminate the species ${ }^{23}$. All phenotypic tests conducted in the present study showed suggestive strains of C. dubliniensis, in addition to the strains confirmed through the molecular test.

The thermotolerance test at $45{ }^{\circ} \mathrm{C}$ is considered easy to perform and is frequently used in association with other phenotypic tests. However, this test presents low specificity according to some authors considering that $C$. albicans may be incorrectly identified as $C$. dubliniensi ${ }^{24}$. In our study, five $(17.2 \%)$ of the isolates did not grow at $45^{\circ} \mathrm{C}$, suggesting the presence of $C$. dubliniensis. Conversely, the two isolates molecularly identified as $C$. dubliniensis grew under this temperature. After studying eight isolates of $C$. dubliniensis, Mähnss et al. ${ }^{22}$ also observed some growth under these conditions. Nevertheless, other authors did not find any strain of C. albicans or C. dubliniensis with unusual behavior ${ }^{25,26}$, as it occurred in the study by Spolidorio et al..$^{27}$, in which all specimens of $C$. dubliniensis were not able to grow at $45^{\circ} \mathrm{C}$.

Aiming to facilitate the isolation and presumptive identification of yeasts, many culture media containing chromogenic substrates have been developed and used to investigate the colonization and infection by C. dubliniensis, considering that the dark green color of its colonies in these media is considered specific for this species ${ }^{28}$.
In this study, four $(13.7 \%)$ isolates showed dark-green color suggestive of $C$. dubliniensis. However, only one of these isolates was confirmed to belong to this species. Sahand et al. ${ }^{29}$ observed that $12(12.2 \%)$ out of the 98 studied strains presented dark-green color, but they were later identified as $C$. albicans ${ }^{29}$. This result differs from that obtained in the study by Kirkpatrick et al. ${ }^{30}$, in which 23 out of 63 strains presented dark-green color, but 16 (69.5\%) were later identified as $C$. dubliniensis.

According to Tintelnot et al. ${ }^{31}$, strains subcultured at $37{ }^{\circ} \mathrm{C}$ in chromogenic medium may lose their ability to show specific coloring. This may explain the mismatch between chromogenic medium coloring and the genotypic results observed in this study.

In recent years, many plant-extract and seed-based culture media have been used as phenotypic markers to distinguish $C$. albicans from C. dubliniensis. Staib agar (or Niger agar) and Tobacco agar are some examples ${ }^{14,15}$.

In the present study, we observed that two (6.9\%) strains presented clamydoconidiums when microcultured in Staib agar, and one of them was molecularly confirmed as $C$. dubliniensis. The other genotypically identified C. dubliniensis strains did not produce such structures. $\mathrm{Al}$ Mosaid et al. ${ }^{32}$ reported a significant percentage of isolates of $C$. dubliniensis (14.6\%) that were not able to produce clamydoconidiums in this medium. According to Tintelnot et al. ${ }^{31}$, the presence of abundant chlamydospore formation is considered to have low predictive value to differentiate these species. However, Pasligh et al. ${ }^{33}$ state that the use of these phenotypic methods, after scanning with chromogenic medium, is a simple, low-cost, highly susceptible technique for differentiation between these species in the laboratorial routine. In our study, the phenotypic technique using Staib agar showed higher susceptibility (96.3\%) in comparison with other techniques.

When cultured in Tobacco agar, six (20.7\%) strains presented abundant formation of clamydoconidiums, characteristics suggestive of $C$. dubliniensis. One of these strains was identified as $C$. dubliniensis using the molecular method, but the other $C$. dubliniensis strains did not present clamydoconidiums in this medium. Notably, Khan et al. ${ }^{15}$ concluded that this medium is $100 \%$ efficient because all $C$. dubliniensis isolates tested had produced abundant clamydoconidiums after incubation. Other authors have also suggested that this is a presumptive method used for

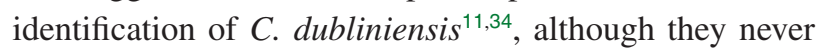
obtained total efficacy in this medium.

In Sabouraud agar medium supplemented with $6.5 \%$ $\mathrm{NaCl}$, five strains did not present any growth. Only two of these strains were identified as $C$. dubliniensis, whereas the others were $C$. albicans. In a study conducted by 
Oliveira et al. ${ }^{35}$, all the 39 strains identified as C. albicans presented growth in this medium; likewise in the study developed by Chowdhary et al. ${ }^{36}$, in which all the 84 strains identified as $C$. albicans presented growth.

In this study, none of the phenotypic tests alone proved to be highly effective for a conclusive identification of C. dubliniensis or C. albicans. No test showed $100 \%$ susceptibility and only the hypertonic medium showed $100 \%$ specificity. In the study performed by Pineda et $a l .{ }^{34}$, despite the fact that none of the tests used was $100 \%$ susceptible or specific, the authors considered that at least three different phenotypic tests should be used to properly distinguish the two species.

The phenotypic techniques used in our study suggested the presence of $C$. dubliniensis among the studied strains. However, there was no coherence between the tests to reach a conclusive identification. Furthermore, none of these methods showed full agreement with the genotypic method.

The results of the identification obtained through phenotypic tests were discussed in relation to the findings of the genotypic identification, which is considered the gold standard.

Therefore, there is still the need to use genotypic methods to achieve conclusive identification and differentiation between species because most of the phenotypic tests show ambiguous results, as demonstrated herein.

\section{ACKNOWLEDGEMENTS}

The authors are grateful to the Fundação de Amparo à Pesquisa do Estado de São Paulo (FAPESP) for the financial support. We would like to thank Prof. Tit. Arnaldo Lopes Colombo for his support in the molecular studies, Prof. Dr. Claudio Mendes Pannuti for his support in the statistical analysis, and Dr. Heloísa Helena de Sousa Marques and Dr. Samantha Andrade for their support with the patients.

\section{CONFLICT OF INTERESTS}

We certify that there is no conflict of interest with any financial organization regarding the material discussed in the manuscript.

\section{REFERENCES}

1. Kadir T, Uygun B, Akyuz S. Prevalence of Candida species in Turkish children: relationship between dietary intake and carriage. Arch Oral Biol. 2005;50:33-7.

2. Domaneschi C, Massarente DB, de Freitas RS, de Sousa Marques HH, Paula CR, Migliari DA, et al. Oral colonization by Candida species in AIDS pediatric patients. Oral Dis. 2011;17:393-8.
3. Sullivan DJ, Westerneng TJ, Haynes KA, Bennett DE, Coleman DC. Candida dubliniensis sp. nov.: phenotypic and molecular characterization of a novel species associated with oral candidosis in HIV-infected individuals. Microbiology. 1995;141:1507-21.

4. Polacheck I, Strahilevitz J, Sullivan D, Donnelly S, Salkin IF, Coleman DC. Recovery of Candida dubliniensis from nonhuman immunodeficiency virus-infected patients in Israel. J Clin Microbiol. 2000;38:170-4.

5. Sullivan DJ, Moran G, Donnelly S, Gee S, Pinjon E, McCartan B, et al. Candida dubliniensis: an update. Rev Iberoamer Micol. 1999;16:72-6.

6. Cimolai N, Davis J, Trombley C. Candida dubliniensis fungemia and vascular access infection. J. Pediatr Hematol Oncol. 2002;24:237-9.

7. Al-Ahmad A, Auschill TM, Dakhel R, Wittmer A, Pelz K, Heumann C, et al. Prevalence of Candida albicans and Candida dubliniensis in caries-free and caries-active children in relation to the oral microbiota - a clinical study. Clin Oral Investig. 2016;20:1963-71.

8. Arikan S, Darka O, Hascelik G, Gunalp A. Identification of Candida dubliniensis strains using heat tolerance tests, morphological characteristics and molecular methods. Mikrobiyol Bul. 2003;37:49-57.

9. Ribeiro PM, Querido SM, Back-Brito GN, Mota AJ, Koga-Ito CY, Jorge AO. Research on Candida dubliniensis in a Brazilian yeast collection obtained from cardiac transplant, tuberculosis, and HIV-positive patients, and evaluation of phenotypic tests using agar screening methods. Diagn Microbiol Infect Dis. 2011;71:81-6.

10. Chavasco JK, Paula CR, Hirata MH, Aleva NA, Melo CE, Gambale W, et al. Molecular identification of Candida dubliniensis isolated from oral lesions of HIV-positive and HIV-negative patients in São Paulo, Brasil. Rev Inst Med Trop S. Paulo. 2006;48:21-6.

11. Bosco-Borgeat ME, Taverna CG, Cordoba S, Isla MG, Murisengo OA, Szusz W, et al. Prevalence of Candida dubliniensis fungemia in Argentina: identification by a novel multiplex PCR and comparison of different phenotypic methods. Mycopathologia. 2011;172:407-14.

12. Kurtzman CP, Fell JW, Boekhout T. The yeasts: a taxonomic study. $5^{\text {th }}$ ed. Amsterdan: Elsevier; 2011.

13. Coleman D, Sullivan D, Harrington B, Haynes K, Henman M, Shanley D, et al. Molecular and phenotypic analysis of Candida dubliniensis: a recently identified species linked with oral candidosis in HIV-infected and AIDS patients. Oral Dis. 1997;3(Suppl 1):S96-101.

14. Staib P, Morschhäuser J. Chlamydospore formation on Staib agar as a species-specific characteristic of Candida dubliniensis. Mycoses. 1999;42:521-4.

15. Khan ZU, Ahmad S, Mokaddas E, Chandy R. Tobacco agar, a new 
medium for differentiating Candida dubliniensis from Candida albicans. J Clin Microbiol. 2004;42:4796-8.

16. Odds FC, Bernaerts R. CHROMagar Candida, a new differential isolation medium for presumptive identification of clinically important Candida species. J Clin Microbiol. 1994;32:1923-9.

17. de Hoog GS, Gerrits van den Ende AH. Molecular diagnostics of clinical strains of filamentous Basidiomycetes. Mycoses. 1998;41:183-9.

18. White TJ, Bruns TD, Lee S, Taylor JW. Amplification and direct sequencing of fungal ribosomal RNA genes for phylogenetics. In: Innis MA, Gelfand DH, Sninsky JJ, White TJ, editors. PCR protocols: a guide to methods and applications. San Diego: Academic Press; 1990. p.315-22.

19. Alves SH, Milan EP, Branchini ML, Nishimura K, Fukushima $\mathrm{K}$, Oliveira LO, et al. First isolation of Candida dubliniensis in Rio Grande do Sul, Brazil. Diagn Microbiol Infect Dis. 2001;39:165-8.

20. Brito Gamboa A, Mendoza M, Fernández A, Díaz E. Detection of Candida dubliniensis in patients with candidiasis in Caracas, Venezuela. Rev Iberoam Micol. 2006;23:81-4.

21. Binolfi A, Biasoli MS, Luque AG, Tosello ME, Magaró HM. High prevalence of oral colonization by Candida dubliniensis in HIV positive patients in Argentina. Medical Mycol. 2005;43:431-7.

22. Mähnss B, Stehr F, Schäfer W, Neuber K. Comparison of standard phenotypic assays with a PCR method to discriminate Candida albicans e Candida dubliniensis. Mycoses. 2005;48:55-61.

23. Marinho SA, Teixeira AB, Santos OS, Cazanova RF, Ferreira CA, Cherubini K, et al. Identification of Candida spp. by phenotypic tests and PCR. Braz J Microbiol. 2010;41:286-94.

24. Campanha NH, Neppelenbroek KH, Spolidorio DM, Spolidorio LC, Pavarina AC. Phenotypic methods and commercial systems for the discrimination between C. albicans and C. dubliniensis. Oral Dis. 2005;11:392-8.

25. Jabra-Rizk MA, Falkler WA Jr, Merz WG, Baqui AA, Kelley JI, Meiller TF. Retrospective identification and characterization of Candida dubliniensis isolates among Candida albicans clinical laboratory isolates from immunodeficiency virus (HIV) infected and non-HIV-infected individuals. J Clin Microbiol. 2000;38:2423-6.

26. Mosca CO, Moragues MD, Llovo J, Al Mosaid A, Coleman DC, Pontón J. Casein agar: a useful medium for differentiating Candida dubliniensis from Candida albicans. J Clin Microbiol. 2003;41:1259-62.
27. Spolidorio DM, Boriollo MF, Estrela C, Spolidorio LC. Diferentes métodos fenotípicos para isolamento e identificação de espécies de Candida. Robrac. 2009;45:18-26.

28. Daef E, Moharram A, Eldin SS, Elsherbiny N, Mohammed M. Evaluation of chromogenic media and seminested PCR in the identification of Candida species. Braz J Microbiol. 2014;45:255-62.

29. Sahand IH, Maza JL, Eraso E, Montejo M, Moragues MD, Aguirre JM, et al. Evaluation of CHROM-Pal medium for the isolation and direct identification of Candida dubliniensis in primary cultures from the oral cavity. J Med Microbiol. 2009;58:1437-42.

30. Kirkpatrick WR, Revankar SG, Mcatee RK, Lopez-Ribot JL, Fothergill AW, Mccarthy DI, et al. Detection of Candida dubliniensis in oropharyngeal samples from human immunodeficiency virus-infected patients in North America by primary CHROMagar candida screening and susceptibility testing of isolates. J Clin Microbiol. 1998;36:3007-12.

31. Tintelnot K, Haase G, Seibold M, Bergmann F, Staemmler M, Franz T, et al. Evaluation of phenotypic markers for selection and identification of Candida dubliniensis. J Clin Microbiol. 2000;38:1599-608.

32. Al Moisaid A, Sullivan D, Salkin IF, Shanley D, Coleman DC. Differentiation of Candida dubliniensis from Candida albicans on staib agar and caffeic-ferric citrate agar. J Clin Microbiol. 2001;39:323-7.

33. Pasligh J, Radecke C, Fleischhacker M, Ruhnke M. Comparison of phenotypic methods for the identification of Candida dubliniensis. J Microbiol Immunol Infect. 2010;43:147-54.

34. Pineda G, Scollo K, Santiso G, Lehmann E, Arechavala A. Aislamiento de Candida dubliniensis en distintos materiales clínicos: análisis de métodos fenotípicos de diferenciación con Candida albicans. Rev Argent Microbiol. 2008;40:211-7.

35. Oliveira VK, Ruiz LS, Oliveira NA, Moreira D, Hahn RC, Melo AS, et al. Fungemia caused by Candida species in a children's public hospital in the city of São Paulo, Brazil: study in the period 2007-2010. Rev Inst Med Trop São Paulo. 2014;56:3015.

36. Chowdhary A, Randhawa HS, Kowshik T, Kathuria S, Roy P, Brandt ME. Application of hypertonic Sabouraud glucose agar for differentiation of Candida dubliniensis from Candida albicans. Diagn Microbiol Infect Dis. 2011;69:440-2. 